\title{
Caring Needs of Cancer Patients from the Perspective of Home Care Nurses: A Qualitative Study
}

\author{
Hadi Hassankhani ${ }^{1}$, Javad Dehghannezhad ${ }^{2 *}$, Azad Rahmani ${ }^{3}$, Mansour \\ Ghafourifard $^{4}$, Amin Soheili ${ }^{5}$, Mojgan Lotfi ${ }^{4}$
}

\begin{abstract}
Objective: Cancer is the third leading cause of death in Iran. Todays, caregiving to patients with cancer is shifting towards home based care, and home care needs from a caregiver's perspective can help improve the patient care. This qualitative study aimed to examine the home care needs of cancer patients from the perspective of home care nurses. Methods: This is a qualitative descriptive study carried out at home care centers in the northwest of Iran. A total of 15 participants were recruited through purposive sampling and underwent face-to-face semi-structured interviews. Data were analyzed through Conventional content analysis method in MAXQDA software. Measures of trustworthiness were established throughout the study using Lincoln and Guba's (1985) criteria (dependability, credibility, transferability, and confirmability). Results: Data analysis resulted in the extraction of four main categories including physical needs (pain relief, gastrointestinal problems including nausea and anorexia and nutritional problems, lethargy, wound care), psychological support (need for hope and emotional support), educational needs (need for information and self-care) and financial support (service insurance coverage, charity support). To promote these patients home care, insurance coverage of nursing home care services was emphasized by the participants. Conclusion: Various aspects of cancer patients home care needs were identified. Interdisciplinary home-based palliative care collaboration is needed to address their physical, psychological, and moral needs.
\end{abstract}

Keywords: Cancer- need- nursing- home care

Asian Pac J Cancer Prev, 23 (1), 71-77

\section{Introduction}

Cancer is the third leading cause of death in Iran. The GLOBOCAN 2020 estimates that there were 19.3 million new cases of cancer and almost 10 million deaths from cancer in 2020 (Sung et al., 2021) Cancer incidence, crude rate, and age-standardized rate (ASR) in Iran increased in 2000-2016 with vast heterogeneity by cancer type, province, and sex (Rajai et al., 2020). new statistics in Iran showed that there were 13,111 new cases of cancer and 79,137 deaths In 2020, and stomach cancer the most commonly diagnosed cancer and the leading cause of cancer death in Iran (Sung et al., 2021), Therefore, the increasing trends in incidence of most common cancers in Iran reinforce the need for the tailored design and implementation of effective national cancer control programs across the country (Roshandel et al., 2021).

Cancer leads to many problems for the patients and their families(Wilson et al., 2016). These issues and problems occur in most aspects of daily life, thereby reducing the quality of life of patients (Mah et al., 2021).
According to the literature review, cancer patients and their families suffer from numerous problems in various aspects such as the cognitive, health care, spiritual, social, emotional, and personal aspects (McCaffrey et al., 2016). The cost of cancer in Iran is significant and is pre-dicted to rise considerably because of the growing new diagnosed cases, which necessitates the implementation of preventive and screening pro-grams in the national cancer control program (Rezapour et al., 2021). It is necessary to develop a comprehensive and holistic care plan for the cancer patients to overcome these problems and improve the quality of life of these patients and their families in various aspects of life (Hui and Bruera, 2016). Daraie et al study showed that the family caregivers were in need of training and support from professionals (Daraie et al., 2021).

In recent years, care for terminally and chronically ill patients has shifted from inpatient centers to the patients' homes (Fabbri et al., 2020). The results of a study showed that most patients with terminally diseases prefer to receive home care and be with their families at 
the end of life (Cai et al., 2021). Furthermore, the studies have shown that home care for terminally ill patients lowers the health system costs, reduces the hospitalization duration. However, home-based palliative care is still not prevalent in the Iranian health system for the chronic and terminally ill patients, including cancer patients (Rassouli and Sajjadi, 2016), and the health system is suffering a shortage of the special units and wards for these patients. Besides, these patients are repeatedly hospitalized on special beds towards the end of their lives (Heydari, 2018). In most cases, hospitalization of these patients does not influence their recovery and imposes heavy costs on the health care systems (Aghabarari and Dehghan Nayeri, 2015), Meanwhile, in many health care systems, cancer care is shifted towards home care (Andersen et al., 2019) and it is necessary to put this necessity on the agenda for health policy-makers on different levels to be able to carry out the required planning (Hassankhani et al., 2018). One of the main components of care planning in this regard is the identification of the care needs of patients with cancer. Therefore, health care professionals, managers, and researchers should value this component to take the necessary measures and provide the necessary facilities accordingly (Wang et al., 2018).

Cancer patients have several unmet care needs and the complexity of the needs increases when cancer care is provided at home (Wang et al., 2018). For planning and providing a high quality home care for cancer patients, the caring needs of these patients should be assessed by health care providers (Puts et al., 2021). A study by Mishra et al., (2020) reported that acute surge of cases and less health care workforce availability during COVID-19 pandemic has shifted the hospital based care model to the home based care model for cancer patients. So, caring the caregivers is an essential part while planning for the care of cancer patients and related policy formulation. Also it is necessary to revise hospitals' discharge program, and home health care center's plan for admission and delivering health care services for cancer patients (Alizadeh et al., 2021). However, few studies have been conducted in Iran on the unsatisfied home care needs of cancer patients and the care needs of these patients are not identified regarding the local and cultural issues. The literature highlights the need to examine the experiences of home care nurses in different cultures, ethnicities, and socioeconomic backgrounds. This qualitative study aimed to examine the home care needs of cancer patients from the perspective of home care nurse

\section{Materials and Methods}

This qualitative study was carried out through conventional content analysis which is used for description of a phenomenon when there are few theories or studies about that phenomenon (Graneheim and Lundman, 2004).

The study settings included the home care counseling centers in East Azerbaijan Province in the northwest of Iran. A purposive sampling technique was used for selection of participants. The inclusion criteria included the nurses who had the experience of working at home care centers and the experience of providing care or management services to cancer patients. The participants were allowed to withdraw the study any time.

The first three participants were selected from the individuals who had rich experiences in providing care to cancer patients and were able to communicate their experiences. After analyzing the interviews, the next participants were selected by the purposive sampling. We tried to obtain a maximum variety of participants by considering the age, gender, and work experience.

Finally, a total of 15 participants took part in this study ( 3 of them were managers of home care centers, 9 participants were nurses with the experience of working with cancer patients at their homes, and 3 participants were among the managers of home care in Tabriz University of Medical Sciences (Table 1).

Data was gathered through semi-structured face-to-face interviews. The interviews were carried out at the time and place agreed upon by the participants. The interviews were carried out with 15 participants in private rooms at the home care centers. The interviews started with the following question: "Please describe your daily

Table 1. Participants' Specifications

\begin{tabular}{lccclc}
\hline No. & Age & Gender & Education & Position & Work experience \\
\hline 1 & 56 & Male & Ph.D. in nursing & Manager of a home care center & 10 \\
2 & 42 & Male & Ph.D. in nursing & Manager of a home care center & 5 \\
3 & 45 & Male & BSN & Manager of a home care center & 10 \\
4 & 51 & Female & MSN & Managers of home care in the university & 16 \\
5 & 37 & Male & BSN & Managers of home care in the university & 13 \\
6 & 32 & Male & BSN & Managers of home care in the university & 8 \\
7 & 54 & Female & BSN & Nurse & 12 \\
8 & 39 & Female & BSN & Nurse & 12 \\
9 & 38 & Male & BSN & Nurse & 8 \\
10 & 43 & Female & BSN & Nurse & 9 \\
11 & 42 & Female & BSN & Nurse & 12 \\
12 & 45 & Male & BSN & Nurse & 11 \\
13 & 32 & Female & BSN & Nurse & 9 \\
14 & 35 & Female & BSN & Nurse & 12 \\
\hline
\end{tabular}


work in the home care settings and what care needs do you meet for cancer patients?" The other questions were asked to access more information and clarify the participants' statements: "What type of care have you so far provided to the cancer patients?" The probing questions were also asked based on the participants' responses, such as the following questions: "Can you elaborate more or give some examples? What did you mean by this statement?" The length of the interviews varied from 35 to 50 minutes and data gathering continued until the data saturation was occurred where no new category emerged from participants' responses. The last two interviews were carried out to ensure data saturation. All interviews were recorded by a digital voice recorder.

Data analysis was conducted using the conventional content analysis method proposed by Lundman and Graneheim (Graneheim and Lundman, 2004). For data analysis, the interviews were transcribed on paper and were then typed into a computer. To gain an overall understanding, the interviews were divided into semantic units. Afterward, the codes were revised several times to be placed in the primary and secondary categories based on their similarities. Finally, after several reviews, the researcher and the participants agreed on certain semantic similarities between the classes. In addition, the researcher attempted to avoid incorporating his assumptions into the data analysis process. MAXQDA 10 software was used for data management.

Four ensuring the rigor of the study, credibility, confirmability, dependability, and transferability were used for data validation.(Graneheim and Lundman, 2004). To ensuring the credibility, there was constant engagement with the research subject and data. Besides, the extracted codes were shared with some of the participants and their opinions were sought (Member checking). To determine data confirmability, an external reviewer familiar with qualitative research checked the research process and findings. To determine the dependability of the findings, all of the activities including the study process and achievement of the findings were recorded carefully, and a report on the research process was provided. To determine data transferability, the research findings were shared with two nurses that did not participate in the study but were in situations similar to the study participants' and their approval was obtained.

This study has been approved by the Research Ethics Committee of Tabriz University of Medical Sciences (IR. TBZMED.REC.1397.675). The information required about this study and voluntary participation in this study was provided to all participants and informed written consent of all participants was obtained to hold and record the interviews. They were also assured that the recorded information was going to be confidential.

\section{Results}

The findings from this study were obtained by interviewing 15 participants ( 7 women and 8 men), 2 of whom had a Ph.D. degree in nursing, one had a master's degree in nursing, and the rest had a bachelor of science in nursing. The average age of the participants was 42.4
2.3 years and their average work experience was 10.8 1.1 years.

Four categories related to the home care needs of cancer patients were identified through the data analysis: physical needs (pain relief, digestive and nutritional issues, fatigue, and wound care), psychological support (the need for hope and the need for affection), educational needs (the need for information and self-care), and financial support (insurance coverage and support from charities).

\section{Physical needs}

The participants voiced the multiplicity of the home care needs of cancer patients. The physical care needs of these patients are mostly met by family caregivers that are not specifically trained, and home care centers are contacted only when specialist care or therapeutic procedures are needed.

\section{Pain relief}

Pain is the most common physical problem in cancer patients. The need for receiving painkillers or the injection of these medications leads to the frequent contact between the family members or the patients and the home care centers. One of the participants said in this regard:

"I went to the house of a patient with metastatic cancer. He was in excruciating pain and he did not know where to get morphine. I told him what to do to get some morphine. The patient was very ill and weak and he was unable to perform his daily chores (Participant. 9)."

\section{Digestive and nutritional problems}

Some other causes of contacting the home care centers were nausea, vomiting, anorexia, severe malnutrition, and the need for the injection of the drugs for these patients. Sometimes the patients' family members contact these centers to request feeding through nasogastric tube (NGT) or parenteral feeding.

"Most cancer patients are unable to eat at home and do not have enough energy. They need the help of nutritionists. Besides, nausea and anorexia are prevalent among them, and when they are unable to eat through the mouth, we administer gavage feeding through nasogastric tube (NGT) or intravenous injections (Participant. 3)".

\section{Wound care}

According to the participants, many cancer patients develop different kinds of skin wounds, especially in the end stages of cancer due to the side effects of radiation therapy or long-term immobility. Due to the lack of specialist, primary care are provided by caregivers and these wounds are mostly reported with delay and the wounds become deeper and more infectious when the home care personnel make a home visit.

"I had a cancer patient who fell into a coma due to an existence of a mass in his head and due to his immobility. He had a grade-3 pressure ulcer that needed special care and home care. The patient also needed care from specialists from different medical fields (Participant. 10)."

\section{Fatigue}

Most of the participants agreed that fatigue, weakness, Asian Pacific Journal of Cancer Prevention, Vol 23 
and severe lethargy are among the other symptoms that make patients contact the home care centers. The participants believed that their severe weakness and fatigue reduced the patients' quality of life significantly. The participants stated that in these cases, they provide training to the patients and their family members to teach them energy saving, and they refer the patients to the appropriate specialists in the complicated cases.

"I was at the bedside of a patient who said: I was an active person but since I have developed the disease I have been feeling tired and bored which is pretty annoying. I do not know what to do to feel energetic again." (Participant. 4)

\section{Psychological support}

The participants stated that psychological support was among the home care needs of cancer patients because most of these patients feel depressed after being diagnosed with cancer. In this case, the patient and their family have to be supported by professional caregivers that are categorized into two sub-categories, namely the need for hope and the need for affection.

\section{Need for hope}

The participants stated that most cancer patients see a terrible future for themselves and doubt on their recovery and on the efficacy of the treatments. However, having the right spirit and hope helps them to fight the disease and recover. Therefore, it is necessary to always raise hope in these patients. One of the nurses stated in this regard as:

"The problem is that when they realize the cancer, they feel broken in spirit and take a hopeless attitude to the treatment. They pay a lot but they think it is useless. They do not know that if they change their lifestyle and expect to recover, they can more easily cope with the disease" (Participant. 3).

\section{The need for affection}

The need for affection is another finding about psychological support, which calls for being compassionate and accepting the patient as a unique individual and valuing their emotions. An analysis of data showed that cancer patients need more emotional support than healthy people. The majority of the participants stated that cancer patients are anxious and feel depressed, bored, and useless. They are also worried about their treatment and the next stages of their disease. Therefore, a person that can properly respond to the patients' emotions and feelings can help the patient.

"I was at a patient's bedside for six months. Early on, I realized the patient was so anxious and upset. He felt bad about many things in his life, and he believed it was over for him. As I managed to finally communicate with him over time and listen to his words, we talked about many issues and we developed a friendship. He found peace and I have been occasionally visiting him as a guest since his recovery" (Participant no. 11).

\section{Educational needs}

Many participants believed that cancer patients receive long-term treatment at home and they must be educated about personal hygiene and the side effects of chemotherapy medications. They also have to provide the related primary care to themselves. These measures contribute to the recovery of the disease and mitigation of the side effects. This category was revealed through two subcategories, namely the need for information and self-care.

\section{Need for information}

This category stresses the need for information about the disease, symptoms, and complications. In this regard, most participants stated that it is necessary to inform the patient about the disease. In some cases, people with a benign cancer are mentally harmed as badly as patients with malignant cancers and their stress can be mitigated by providing the necessary information. Regarding the side effects of the chemotherapy, if patients receive the required education, they can cope with chemotherapy more easily and will experience lower levels of stress. One of the nurses said in this regard:

"Many people think cancer is a giant monster and they are devoid of awareness about it. They believe that cancer is equivalent to death. They are terrified of chemotherapy and believe it is an extremely difficult process. There is no awareness about this issue and they are always confused and distressed" (Participant no. 6).

\section{Self-care needs}

Another sub-category of the educational need of cancer patients is self-care needs. As a self-care agent, the patient plays a substantial role in controlling and managing the disease. The enforcement of certain medical tips and advice (e.g. personal hygiene, timely intake of medications, the right diet, and the required physical activities) contributes to the treatment of the disease and reduces its complications.

"Some patients wear a mask everywhere they go but it is not a good thing because they feel isolated and the need for social communication is very important. Some people do not wear a mask at all and they do not know how to keep their distance from others and keep their hands clean. Many people also do not know the right diet for them. These things must be taught" (Participant. 5).

\section{Need for financial support}

Financial support is one of the primary categories identified in this study, which reflects the need for financial support in cancer patients. According to our analyses of the data obtained from the interviews, financial support must be one of the priorities in the health system for cancer patients because the treatment and medication costs for these patients are extremely heavy and the duration of treatment of these patients is long. Most of these patients come from the low-income class, which includes two sub-categories of needs: the need for the insurance coverage of services and the need for support from charities. 


\section{Need for insurance coverage of services}

Most of the participants stated that the need for the insurance coverage of home nursing care services is essential for cancer patients, and stated that since providing home care services is highly expensive and the patient has to pay all of the costs, insurance companies should pay part of the expenses for the home care services to encourage the patients to use these centers. Most nurses also believed that insurance coverage of services is one of the important priorities for these patients, which prevents their frequent referrals and hospitalizations. In this regard, one of the nurses said:

"Many patients cannot afford the expenses and they pay for all the services they receive at home. Even the insurance companies do not pay part of the costs. The patient and their family are emotionally destroyed and they watch their loved ones pass away while they cannot do anything about it. It is very difficult for them (participant no. 2)."

\section{Need for support from charities}

According to the participants, most cancer patients need the help of charities to support them financially, meet their treatment needs and pay for their expenses. However, many home care nurses acknowledged that there is no link between the identification of low-income patients and making arrangements with charities for cancer patients. One of the nurses also said in this regard:

"I had a patient that was the father of a family, and he could not go to work because of his illness. He also had a child. I only managed to find a charity and the charity only accepted to pay for the medications (Participant. 7)."

\section{Discussion}

The present study was an attempt to study the home care needs of cancer patients from the viewpoint of home care nurses. The results of the data analysis showed that these needs are classified into four categories of the physical, psychological, educational and financial needs.

A review study by Wang et al., (2014) on the unsatisfied needs of cancer patients showed that these patients have several needs such as physical, financial, social, and spiritual needs (Kang et al., 2014), which is in line with the findings from the present study that include the various aspects of these needs.

The participants in this study believed that cancer patients have some physical needs such as pain, digestive problems including nausea and anorexia, nutritional problems, lethargy, and pressure ulcer. In this regard, a review study referred to the unmet physical needs of cancer patients including digestive problems and fatigue(Kotronoulas et al., 2017). A study by Kang et al., (2014) in Korea reported that anorexia is one of the most common symptoms of cancer patients, which was managed by home-based care services (Woo et al., 2015). Kotronoulas et al., (2017) introduced nutritional problems as one of the main needs of cancer patients that receive home care and suggested that providing information about this issue is among the needs of these patients. Moreover, the findings of a study in Canada indicated that wound care is one of the important needs of cancer patients, which has to be performed coherently in collaboration with the care team and the family to take care of the patient's wound (Woo et al., 2015).

Most of the participants believed that psychological support was one of the home care needs of cancer patients. This finding is in line with other studies that introduce psychological support for cancer patients and their families as the needs of these patients (Schenker et al., 2014; Movahedi et al., 2015; Rachakonda et al., 2015). One of the other needs is the patient's hope. The participants stated that these patients mostly lose their hope and it is necessary to determine the attainable goals in this regard along with the roads to the attainment of these goals to be able to motivate the patient to put hope in the attainment of those goals. Movahedi et al., (2015) showed that patients' hope could be fostered in cancer patients through care interventions and hope therapy, which improves life expectancy and overall health. The research findings also showed that another sub-category of psychological support is emotional support for the patients and most participants reported a considerable need for emotional support because of their complicated disease and the difficult treatment requirements including chemotherapy, hence the need for sensitivity to patients' emotions and feelings.Vivar and McQueen (2005) also reported that cancer patients have unsatisfied emotional needs and a person that understands their feelings needs to be by their side or the patients should be classified into peer groups to establish the necessary emotional connections.

The need for education is one of the primary categories identified through the research findings, which is among the needs of cancer patients, and the participants believe that these patients lack awareness about the disease and its complications as well as the methods of self-care. Ansari et al., (2018) indicated that one of the educational needs of cancer patients is the empowerment of the patients and their caregivers, which is in line with the findings from this study (Ansari et al., 2018). According to the findings, the need for information about cancer and its complications is among the educational needs of cancer patients. Khoshnoud et al., (2019) indicated that cancer patients are not adequately aware of the nature of the disease and they have to be informed about the proper control of the disease and the cancer treatment methods. Another finding was the need to educate the patient about self-care and independence. In this regard, a review study showed that having independence and the ability to practice self-care are among the unsatisfied needs of cancer patients that must be reinforced (Effendy et al., 2015).

Financial support was one of the categories identified through the findings from this study, and the participants believed that most cancer patients are in the low-income social class and they need to be supported by government organizations and charities to receive treatment and self-care facilities. Effendy et al., (2015) showed that financial support is one of the unmet needs of cancer patients in most Asian countries they argued that financial problems are among the most prevalent problems of these patients. One of the factors involved in the financial support for nursing care services is the insurance coverage

Asian Pacific Journal of Cancer Prevention, Vol 23 
and the participants in this study stated that home care services are not covered by any insurance company. Besides, most patients are not supported by any voluntary organization including the charities. A study in Florida revealed that insurance coverage is among the financial needs of the survivors of cancer patients and financial support, including having an adequate income, is among the needs of these patients (Burg et al., 2015).

The findings from this study provide a deep understanding and insight into the viewpoints of home care nurses about the care needs of cancer patients and reveal the home care needs of cancer patients. Policy-makers can make plans for cancer patients based on the study findings within the scope of home care services. Furthermore, since this study is the first qualitative study of the home care needs of cancer patients from the viewpoint of home care nurses in the cultural context of Iran, its results can reveal the different aspects of the care needs of cancer patients.

In the current research, nurses were the only type of health care practitioner interviewed and the experiences of other health personnel were not taken into account. Research to investigate the perspectives of other health personnel, such as physicians nursing assistants, and others may bring further insight to home care needs of cancer patients.

The results of the present study identified the different aspects of the care needs of cancer patients. In addition to their physical and educational problems, cancer patients have the need for psychological support and interdisciplinary cooperation to value the different aspects of this need. It is also suggested that the provision of care to these patients has to be put on the agenda for the authorities in different palliative care models to improve the quality of care provided to these patients by meeting these needs and contributing to the life quality of cancer patients.

\section{Author Contribution Statement}

The authors confirm contribution to the paper as follows: study conception and design: G, D AND H, H; data collection: A, R; analysis and interpretation of results: M, L AND A.S Author, draft manuscript preparation: M, GH. All authors reviewed the results and approved the final version of the manuscript.

\section{Acknowledgements}

This study was approved by the Regional Research Ethic Committee at the Tabriz University.

Thesis data of javad dehghannezhad with title Designing and implementing a home-based palliative care program in cancer patients: a mix method study of action research.

\section{Funding statement}

This work was supported by Tabriz University of Medical sciences.

\section{Conflict of interest}

All authors have no conflicts of interest.

\section{References}

Aghabarari M, Dehghan Nayeri N (2015). Futile Care: challenges of applying futility concept in caring domain. J Hayat, 21, 1-5.

Alizadeh Z, Rohani C, Rassouli M, et al (2021). Transitional Cancer Care Program from Hospital to Home in the Health Care System of Iran. Asian Pac J Cancer Prev, 22, 1231-7.

Andersen SK, Croxford R, Earle CC, et al (2019). Days at home in the last 6 months of life: a Patient-Determined quality indicator for cancer care. J Oncol Pract, 15, e308-e15.

Ansari M, Rassouli M, Akbari ME, et al (2018). Educational needs on palliative care for cancer patients in Iran: A SWOT analysis. Int J Commun Based Nurs Midwifery, 6, 111.

Burg MA, Adorno G, Lopez ED, et al (2015). Current unmet needs of cancer survivors: Analysis of open-ended responses to the A merican $\mathrm{C}$ ancer $\mathrm{S}$ ociety $\mathrm{S}$ tudy of $\mathrm{C}$ ancer $\mathrm{S}$ urvivors II. Cancer, 121, 623-30.

Cai J, Zhang L, Guerriere D, et al (2021). Where do cancer patients in receipt of home-based palliative care prefer to die and what are the determinants of a preference for a home death?. Int J Environ Res Public Health, 18, 235.

Daraie S, Hasanvand S, Goudarzi F, et al (2021). Gaining experience over time: The family caregivers' perception of patients with a tracheostomy in home care. Iran J Nurs Midwifery Res, 26, 137.

Effendy C, Vissers K, Osse BH, et al (2015). Comparison of problems and unmet needs of patients with advanced cancer in a European country and an Asian country. Pain Pract, 15, 433-40.

Fabbri M, Caldas AC, Ramos JB, et al (2020). Moving towards home-based community-centred integrated care in Parkinson's disease. Parkinsonism Related Disorders, 78, 21-6.

Graneheim UH, Lundman B (2004). Qualitative content analysis in nursing research: concepts, procedures and measures to achieve trustworthiness. Nurs Edu Today, 24, 105-12.

Hassankhani H, Rahmani A, Dehghannezhad J (2018). "Letter to Editor" development of palliative care in cancer patients: A Neglected Necessity. Iran J Nurs, 31, 1-5.

Heydari H (2018). Home-based palliative care: A missing link to patients' care in Iran. J Hayat, 24, 97-101.

Hui D, Bruera E (2016). Integrating palliative care into the trajectory of cancer care. Nat Rev Clin Oncol, 13, 159.

Kang MH, Moon YS, Lee YJ, et al (2014). Physical symptoms and psychiatric, social, spiritual and economical care needs of patients under home-based cancer service. Korean $J$ Hospice Palliative Care, 17, 216-22.

Khoshnood Z, Dehghan M, Iranmanesh S, et al (2019). Informational needs of patients with cancer: a qualitative content analysis. Asian Pac J Cancer Prev, 20, 557.

Kotronoulas G, Papadopoulou C, Burns-Cunningham K, et al (2017). A systematic review of the supportive care needs of people living with and beyond cancer of the colon and/or rectum. Eur J Oncol Nurs, 29, 60-70.

Mah K, Chow B, Swami N, et al (2021). Early palliative care and quality of dying and death in patients with advanced cancer. BMJ Supportive Palliative Care, 2021.

McCaffrey N, Bradley S, Ratcliffe J, et al (2016). What aspects of quality of life are important from palliative care patients' perspectives? A systematic review of qualitative research. J Pain Sympt Manage, 52, 318-28. e5.

Mishra S, Biswas S, More S, et al (2020). Caring the cancer caregivers in the Era of COVID-19 outbreak. Asian Pac J Cancer Care, 5, 203-8.

Movahedi M, Movahedi Y, Farhadi A (2015). Effect of hope therapy training on life expectancy and general health in 
cancer patients. J Holistic Nurs Midwifery, 25, 84-92.

Puts M, Szumacher E, Dawe D, et al (2021). Never too old to learn new tricks: Surveying Canadian healthcare professionals about learning needs in caring for older adults with cancer. J Geriat Oncol, 12, 262-73.

Rachakonda K, Mathew George MS, Oldmeadow C (2015). Unmet supportive Cancer care needs: an exploratory quantitative study in rural Australia. World J Oncol, 6, 387.

Rajai N, Ghanbari A, Yoosefi M, et al (2020). National and subnational trends in incidence and mortality of lung cancer in Iran from 1990 to 2016. Asia Pac J Clin Oncol, 16, 129-36.

Rassouli M, Sajjadi M (2016). Palliative care in Iran: Moving toward the development of palliative care for cancer. $\mathrm{Am} \mathrm{J}$ Hospice Palliative Med, 33, 240-4.

Rezapour A, Nargesi S, Mezginejad F, et al (2021). The economic burden of cancer in iran during 1995-2019: A systematic review. Iran J Public Health, 50, 35-45.

Roshandel G, Ferlay J, Ghanbari-Motlagh A, et al (2021). Cancer in Iran 2008 to 2025: Recent incidence trends and short-term predictions of the future burden. Int J Cancer, 2021.

Schenker Y, Park SY, Maciasz R, et al (2014). Do patients with advanced cancer and unmet palliative care needs have an interest in receiving palliative care services?. $J$ Palliative Med, 17, 667-72.

Sung H, Ferlay J, Siegel RL, et al (2021). Global cancer statistics 2020: GLOBOCAN estimates of incidence and mortality worldwide for 36 cancers in 185 countries. CA Cancer J Clin, 71, 209-49.

Vivar CG, McQueen A (2005). Informational and emotional needs of long-term survivors of breast cancer. $J A d v$ Nurs, 51, 520-8.

Wang T, Molassiotis A, Chung BPM, et al (2018). Unmet care needs of advanced cancer patients and their informal caregivers: a systematic review. BMC Palliative Care, 17, 1-29.

Wilson O, Avalos G, Dowling M (2016). Knowledge of palliative care and attitudes towards nursing the dying patient. $\mathrm{Br} J$ Nurs, 25, 600-5.

Woo KY, Krasner DL, Kennedy B, et al (2015). Palliative wound care management strategies for palliative patients and their circles of care. Adv Skin Wound Care, 28, 130-40.

\section{c) (7) (8)}

This work is licensed under a Creative Commons AttributionNon Commercial 4.0 International License. 